\title{
What Does the Future Hold for Surgical Breast Specialists? Implications for Breast Fellowship Training
}

\author{
Scott H. Kurtzman, MD FACS \\ Waterbury Hospital - Surgery, Waterbury, CT
}

Surgeons have long demonstrated an interest in specialized training in breast diseases. In 2002, the Society of Surgical Oncology (SSO), The American Society of Breast Diseases (ASBD), and the American Society of Breast Surgeons (ASBrS) collaborated to create an accreditation process for breast fellowship training. The process was designed to accommodate physicians from any discipline to receive multidisciplinary training in the care of patients with breast problems. Site visits to known breast fellowship programs began shortly thereafter as a collaborative effort of the three groups. The majority of physicians who have sought this training have been surgeons.

The program has been quite successful. Currently there are more than 47 approved programs that graduate 64 fellows every year. These physicians are well trained to take care of patients with breast concerns. There is considerable demand from the public for physicians whose area of concentration is in breast diseases. Hospitals, practices, and breast centers have actively recruited these well-qualified doctors. They will form the foundation for the next generation of leaders in breast surgery. Many of these fellowship-trained surgeons choose to restrict their practice to breast diseases.

However, the world is changing. Tremendous advances in imaging, biopsy techniques, genetic analyses, and adjuvant and neoadjuvant chemotherapy and hormonal therapies have dramatically altered the landscape. Even

Dr. Kurtzman is the former Chair of the Society of Surgical Oncology Training Committee and conducted the first approvals of the SSO/ ASBrS/ASBD Breast Fellowships.

(C) Society of Surgical Oncology 2016

First Received: 15 March 2016;

Published Online: 24 June 2016

S. H. Kurtzman, MD FACS

e-mail: skurtzman@wtbyhosp.org more effective diagnostics, prognostics, and treatment options are on, not beyond, the current horizon. Additionally, the payment structure for physicians and hospitals is rapidly changing.

It would not be hard to take the most nihilistic approach and argue that the current training and practice patterns of these new fellows will leave them in a position where it will be increasingly difficult to sustain their income. Other breast specialists will quickly be able to do almost everything that is currently in the areas covered by breast surgeons.

Radiologists dominate screening and diagnostic imaging. In many hospitals, radiologists and surgeons cooperate in sharing image-guided biopsy; in others, the sharing is done grudgingly or does not occur at all. It is fair to say that most radiologists are at least as capable as most surgeons in performing image-guided biopsies. Since they control the gateway to image-detected cancers, i.e. mammography and magnetic resonance imaging (MRI), the opportunity and need for surgeons is unclear and in jeopardy. Furthermore, open surgical biopsy is almost never needed and certainly cannot sustain a practice. In the US, the percentage of patients undergoing open surgical biopsy has declined from more than $75 \%$ in the 1990 s to less than $20 \%$ in more contemporary series. ${ }^{1}$ The National Accreditation Program for Breast Centers (NAPBC) Standard 2.9 states that "Palpation-guided or image-guided needle biopsy is the initial diagnostic approach rather than open biopsy." While not all patients can have image-guided biopsies, the goal is somewhere around $90 \%{ }^{2}$

Approximately $4-14 \%$ of patients who have an imageguided vacuum-assisted biopsy receive a diagnosis of atypical hyperplasia., ${ }^{3,4}$ These patients now undergo open surgical biopsy. It is not hard to imagine that with advances in tumor genetics and profiling we will prove that the need for open surgical biopsy after a diagnosis of atypical hyperplasia by core biopsy is even more limited. Newer 
biopsy techniques and more intricate non-histologic analyses will give us the information that was once only possible with open operations.

We have made remarkable advances in chemotherapy and hormonal therapy, and the majority of even advanced cancers respond to neoadjuvant treatments. Advances in genomics and proteomics will certainly provide even better tools to describe personalized and more effective treatments that will drive down the need for surgical treatment of many breast cancers. After neoadjuvant therapy, small residual cancers may someday be treated with ablative percutaneous techniques that will be within the expertise of non-surgeons. Similarly, it is likely that we will discover which non-invasive breast diseases even need to be treated, further limiting the number of patients who will need breast operations.

As we come to better understand the genetics of breast cancer we will identify more women who stand to benefit from prophylactic mastectomies. While breast surgeons now do most of these operations, most of them do not do their own reconstructive operations. While it is comfortable to argue that breast surgeons are the most qualified to do these operations, that argument is self-serving and probably not valid. Most plastic surgeons who now control the majority of breast reconstructive operations are already doing breast reduction operations. It is hard to argue that they are not capable of performing prophylactic mastectomies.

While it can be fairly stated that surgeons are currently the first clinicians who see patients with breast disorders, it is not hard to envision other non-surgical or even nonphysician practitioners guiding patients into the system. As surgeons we feel that we are in the best position to guide patients from diagnosis through treatment; however, without the need to perform procedures, it will be hard, from a fiscal point of view, to maintain a viable practice.
The current payment system is designed to reward procedures, not cognitive aspects of care.

Breast specialists who have restricted their practice will face many obstacles if they find the need to return to general surgery. Credentialing Committees and Surgery Department Chairs (if they even exist in the future) will be understandably hesitant to allow a surgeon to take general surgery or trauma call after 10 years of a breast-only practice.

For all of these reasons we need to start planning the future of the surgical breast specialists. There may be a real danger that fellows who are training in 2016 will have a hard time paying their bills in 2026. We need to start considering how we can preserve the positive attributes of breast specialists in the quickly changing new future. Solutions that focus on counseling, physical examination, and guidance of breast patients will be inadequate. It is time to start a dialogue about where this area of specialization is headed.

\section{REFERENCES}

1. Williams RT, Yao K, Stewart AK, Winchester DJ, Turk M, Gorchow A, et al. Needle versus excisional biopsy for noninvasive and invasive breast cancer: report from the National Cancer Data Base, 2003-2008. Ann Surg Oncol. 2011;18(13):3802-10.

2. Calhoun KE, Anderson BO. Needle biopsy for breast cancer diagnosis: a quality metric for breast surgical practice. J Clin Oncol. 2014;32(21):2191-2.

3. Winchester DJ, Bernstein JR, Jeske JM, Nicholson MH, Hahn EA, Goldschmidt RA, et al. Upstaging of atypical ductal hyperplasia after vacuum-assisted 11-gauge stereotactic core needle biopsy. Arch Surg. 2003;138(6):619-22, discussion 622-3.

4. Eby PR, Ochsner JE, DeMartini WB, Allison KH, Peacock S, Lehman CD. Is surgical excision necessary for focal atypical ductal hyperplasia found at stereotactic vacuum-assisted breast biopsy? Ann Surg Oncol. 2008;15(11):3232-8. 